\title{
A Multiple Antenna System Design for Wearable Device Using Theory of Characteristic Mode
}

\author{
J. Chen ${ }^{1}$, M. Berg ${ }^{1}$, V. Somero ${ }^{2}$, H. Y. Amin ${ }^{1}$ and A. Pärssinen ${ }^{1}$ \\ ${ }^{1}$ Center for Wireless Communications, University of Oulu, Oulu, Finland, Jiangcheng.chen@oulu.fi \\ ${ }^{2}$ Polar Electro Oy, Oulu, Finland
}

\begin{abstract}
In this paper, a wearable three-antenna system aiming for cellular Internet of Things (IoT) applications is proposed. The presented antenna system consists of two cellular antennas (LTE low- and mid-band antennas) and one GPS antenna designed by using the Theory of Characteristic Mode (TCM). Both the simulated and measured results with good agreements are provided in this work. As a result of successful excitation of chassis modes, the proposed cellular antennas can cover the desired cellular bands between $699-862$ $\mathrm{MHz}$ and 1710-2155 $\mathrm{MHz}$ with corresponding wideband matching circuits. Meanwhile, the proposed GPS antenna operates with a good right hand circular polarization (RHCP) performance (at least $5 \mathrm{~dB}$ higher gain than left hand circular polarization (LHCP)). Reasonable isolation performances among three antennas are also gained due to utilization of orthogonal modes. The results also show that with the proximity of human body (wrist phantom), over the wideband operation bands all antennas can achieve good efficiency. The averaged levels of $-10 \mathrm{~dB},-6 \mathrm{~dB}$, and $-4 \mathrm{~dB}$, respectively for LTE low band, mid band and GPS band are achieved, indicating its potential in future wearable cellular IoT applications.
\end{abstract}

Index Terms-wearable antenna, Theory of Characteristic Mode (TCM), small mobile terminal antenna, wristband antenna

\section{INTRODUCTION}

In these days, Long Term Evolution (LTE) is evolving with two new narrowband technologies: eMTC (enhanced Machine Type Communication, often referred to as LTE-M) and NB-IoT (Narrow Band-Internet of Things) that will pave the path to $5 \mathrm{G}$ [1]. The evolution of LTE for LTE-M and NB-IoT, in turn, will enable Cellular IoT for low cost, low power and wide area deployments [2]. Moreover, IoT devices are able to use PAN, LAN, WAN or cellular networks, and hence numerous services are envisioned for cellular IoT. Therefore, it can be seen that the market of smart mobile and wearable devices, e.g. smart watch which is a good candidate, are rapidly increasing and have attracted great attention from industries and publics. Since smart wearable devices need the access to wireless data networks (Wireless LAN or cellular systems), antenna becomes an indispensable component.

Conventional mobile terminal antennas such as loop antenna [3], Inverted-F antenna (IFA) [4], monopole antenna [5] and slot antenna [6] are used in smartwatches for 2.4$\mathrm{GHz}$ Wi-Fi or Bluetooth (BT) applications. However, since large electrical length is required in LTE sub-GHz bands, it is difficult to install the low band antenna into the watch's casing due to the limited physical dimension of the casing. In addition, the bandwidth of a radiator is limited by high quality factor $\mathrm{Q}$ of the small physical dimension according to [7] and [8]. Although in our unpublished paper [9], a narrow band IFA mounted on the casing is proposed for LTE Band $20 \mathrm{UL}(832-862 \mathrm{MHz})$, an active component, namely RF switch, is further needed to make the antenna tunable so that it can cover all other low operation bands. In turn, the total efficiency performance will be degraded by the insertion loss of the active component. Considering the physical dimension and performance of bandwidth and total efficiency, the abovementioned antenna designs, therefore, are not very attractive in low frequency cellular bands.

To implement the low band applications, some antenna designs based on wristband are proposed in [10], [11] and [12]. Nevertheless, the worst total efficiency level of the metal belt dipole and monopole antennas in [10] drops to below $-13 \mathrm{~dB}$ and $-10 \mathrm{~dB}$ for LTE low band and high band respectively, which means degraded link performance higher current drain of the power amplifier. The Planar Inverted-F antennas (PIFAs) on wristband in [11] are of such thickness $(6 \mathrm{~mm})$ that they are impractical in commercial products. Also, the bandwidth is too narrow to meet the desired operation bands of wideband cellular communication (LTE low band: 699-862 MHz and mid-band: 1710-2155 MHz). Despite well achieved bandwidth performance by a metal watch strap antenna in [12], one of the most important figure of merits, total efficiency, was not investigated. Thus, these wristband based antenna solutions are less likely to meet requirements of cellular IoT applications.

In this paper, a wearable antenna system consisting of LTE low band, mid-band and GPS antennas is presented. The following simulations are performed by CST Microwave Studio (MWS) co-simulated with CST Design Studio (DS) and Optenni Lab. Specifically, LTE low band antenna is designed based on the Theory of Characteristic Mode (TCM) and a capacitive coupling element (CCE) is used to excite the fundamental chassis mode of the device. To increase the electrical length, the ground of the chassis is extended to the wristband of the smartwatch. Meanwhile, two IFAs locating at top edges of the watch casing are employed for GPS and LTE mid-band. The performances in terms of matching, efficiency and radiation pattern are investigated. Moreover, a 
matching circuit for each antenna is also added to achieve wideband matching performance. Both, simulated and measured results, are given and discussed.

\section{ANTENNA DESIGN AND CONFIGURATION FOR SMARTWATCH}

Characteristic Mode Analysis (CMA) is performed to provide a physical insight into the resonant behavior of a chassis to examine current modes it can support. As studied in [13], strong characteristic electric field distributions of the fundamental mode occur at two short edges of the mobile terminal chassis, especially at corners, while it is very weak at the center of the chassis. Given that, the fundamental mode of the chassis can be efficiently excited by employing a voltage source (e.g. a PIFA in [13], a monopole antenna or CCE in [14] and [15]) at the edge or corner, enhancing the performance of bandwidth and radiation efficiency.

It can be seen from Fig. 1 that the proposed chassis of the smartwatch includes a planar part (the PCB of smartwatch) connected by a very narrow metal tab $\left(1.8 \times 3 \mathrm{~mm}^{2}\right)$ at the center of chassis to a bent part on wristband (ground extension of the PCB with a thickness of zero), where the gap between the planar chassis and bent strap is $1.8 \mathrm{~mm}$. Two long slots at roughly the center of the chassis increase the current path of fundamental mode and hence increase the effective electrical length. In particular, the design of the small tab connection is to ease the installation complexity of product in reality and make it more practical. The characteristic mode analysis is carried out and shown in Fig. 2. Characteristic angles of the first six characteristic modes are presented in Fig. 2(a) and it can be seen that the first two resonances (the first and third characteristic modes) are at 1.4 $\mathrm{GHz}$ and $3.6 \mathrm{GHz}$ respectively according to the fact that the resonance takes place when the characteristic angle equals to $180^{\circ}$ [13]. The electrical field distributions of the first and third characteristic modes at their respective resonant frequency are shown in Fig. 2(b) and (c). Similar to [13], [14] and [15], the fundamental mode has stronger electrical field distribution at edges, especially at corners (see Fig. 2(b)), indicating the optimal feeding position of a voltage source. As observed in Fig. 2(c), the higher characteristic mode at $3.6 \mathrm{GHz}$ has two electrical field distribution nulls along the chassis length whereas three strong electrical field distributions occur at two edges and the center of the entire chassis. Again, the location with strong electrical field distribution reveals the optimal feeding positon for higher resonance excitation.

Concerning the physical dimension limitation of the watch, a CCE is applied to excite the fundamental mode, implementing the LTE low band antenna (ANT1). Meanwhile, two IFAs for LTE mid-band antenna (ANT2) and GPS antenna (ANT3) are also proposed and all the necessary dimensions are provided, see Fig. 3(a), (b) and (c). The three proposed antennas are mounted on the top edge of the planar part of the chassis, which is installed into an ABS plastic casing with a permittivity of 2.7 and a loss tangent of 0.005. The bent part is placed on the surface of the rubber wristband with a permittivity of 3 . A wrist phantom with a radius of $31.5 \mathrm{~mm}$ is also introduced underneath the casing to evaluate the influence of human body. The phantom is set as a dispersive model based on CTIA Certification Standard [16].

As mentioned in [14], the CCE itself is a non-resonant element and, in general, it is naturally poorly matched. Thus a matching circuit is a necessity for this kind of antenna. In addition, matching circuits for ANT2 and ANT3 are also added, where the matching circuit of ANT2 is for wideband matching purpose whilst the matching circuit of ANT3 is for enhancing the isolation with ANT2. In this work, the matching circuit layout is included in the 3D model of the simulation and lumped matching components are presented by ports in CST MWS shown in Fig. 3(c), which are cosimulated with CST DS to design the matching circuits. Each antenna is fed by a coplanar waveguide (CPW) transmission line. The matching networks made by ideal components in

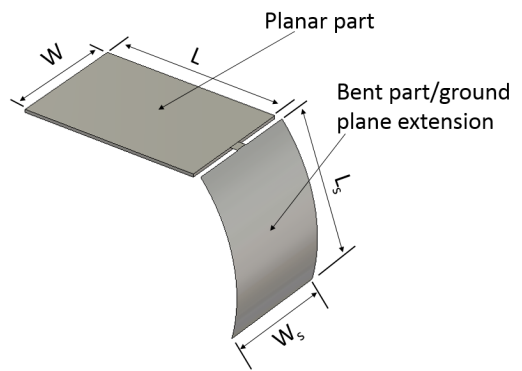

Fig. 1. Overview of the smartwatch chassis. The dimensions are: $\mathrm{L}=45$ $\mathrm{mm}, \mathrm{L}_{\mathrm{s}}=40 \mathrm{~mm}, \mathrm{~W}=30 \mathrm{~mm}$ and $\mathrm{W}_{\mathrm{s}}=30 \mathrm{~mm}$. Thickness of planar chassis is $0.8 \mathrm{~mm}$. The structure is modelled as PEC.

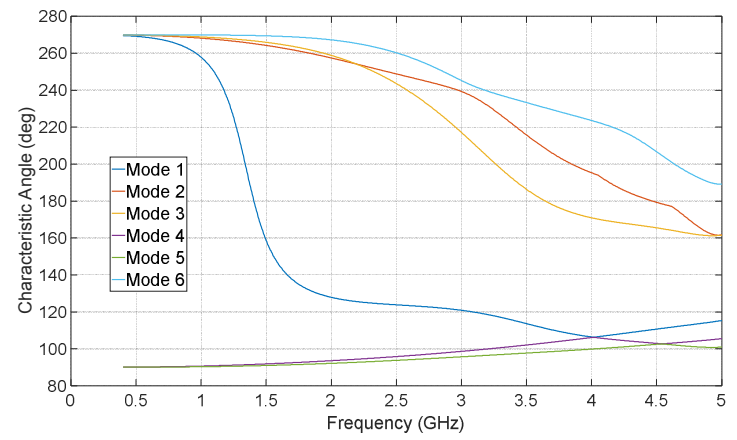

(a)
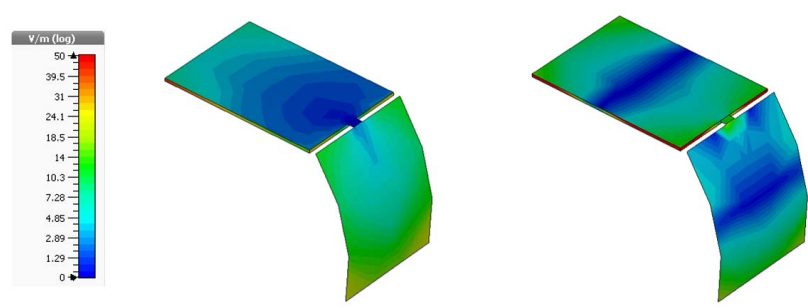

(b)

(c)

Fig. 2. (a) Characteristic angles of first 6 modes of the proposed chassis; The electrical field distributions of (b) the first charactristic mode at 1.4 $\mathrm{GHz}$ and (c) the third charactristic mode at $3.6 \mathrm{GHz}$. 


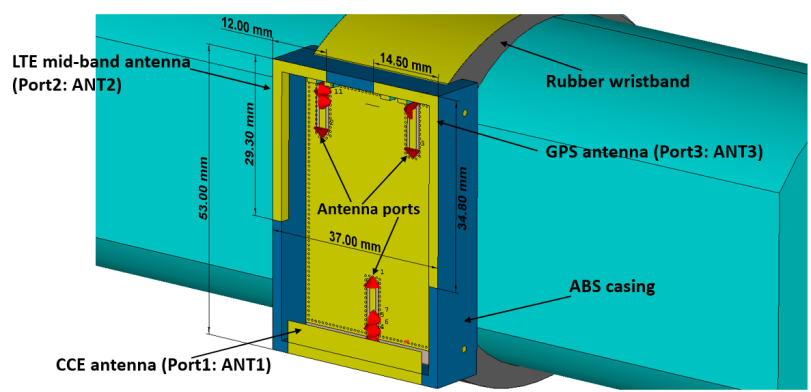

(a)

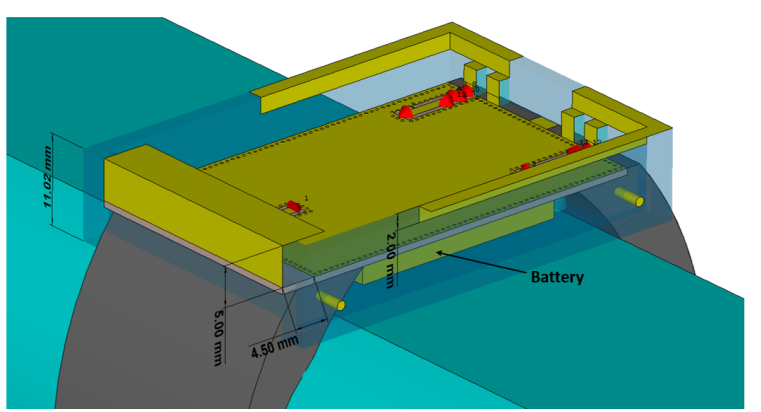

(b)

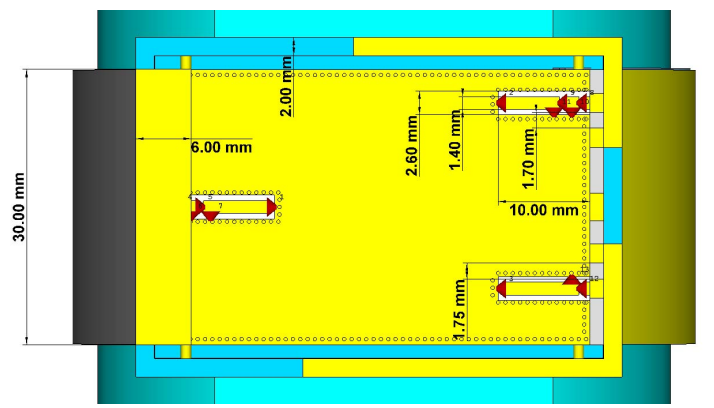

(c)

Fig. 3. Configuration of the three-antenna system. The dimensions for all elements are shown in (a), (b) and (c).

CST DS corresponding to the matching layout in CST MWS are shown in Fig. 4(a) and for clarity the matching topology of each antenna is revealed in Fig. 4(b). Furthermore, the lumped elements in simulation are ideal components whilst Murata's high $\mathrm{Q}$ series of RF inductor and capacitor are employed in prototype. Both, matching component values in the simulation and prototype, based on Fig. 4(b), are given in TABLE I. It can be seen that component values in prototype agree well with those in the simulation, except for some modifications in matching circuit of ANT2 to achieve better wideband matching behavior in practice.

The prototype of the proposed antenna system for wearable application and its installation in Satimo StarLab chamber are shown in Fig. 5(a) and (b) respectively. In particular, the ABS plastic casing is fabricated by Stratasys 3D printer. ANT1 is made up of a folded brass sheet (with a conductivity of $2.74 \mathrm{e}+007 \mathrm{~S} / \mathrm{m}$ ) whereas ANT2 and ANT3 are made of copper tape $(5.96 \mathrm{e}+007 \mathrm{~S} / \mathrm{m})$. To reduce the effect of surface current three feeding coaxial cables are placed in the center of the chassis and a ferrite is applied to these three cables on the back of the casing.

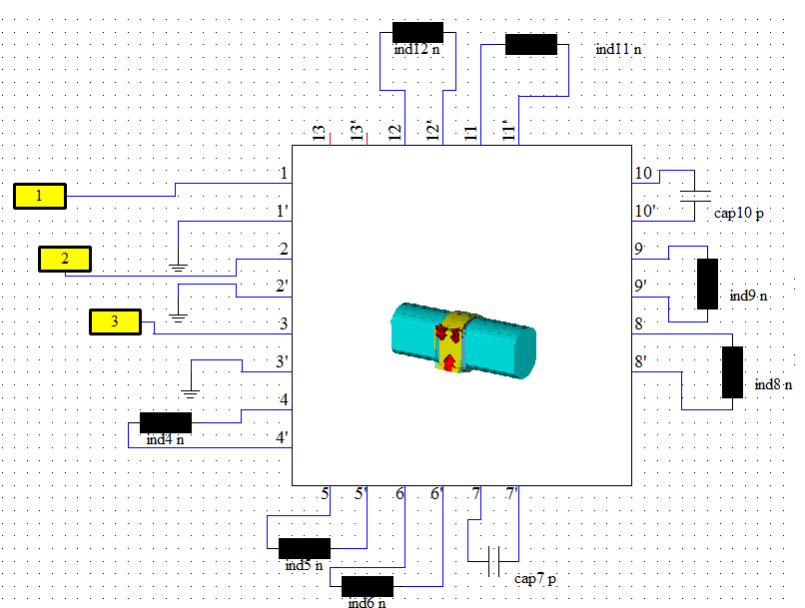

(a)
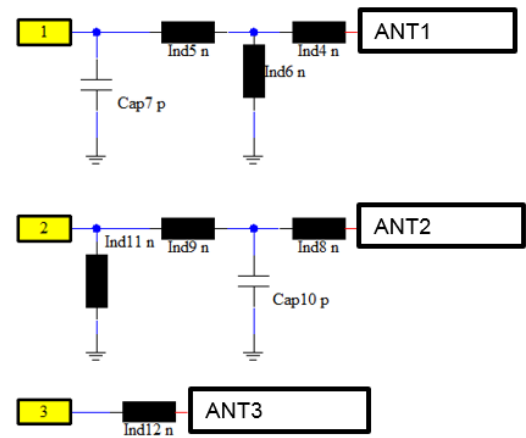

(b)

Fig. 4. (a) Matching networks in CST DS; (b) Matching topology for each antenna.

TABLE I. COMPONENTS VALUES IN SIMULATION AND PROTOTYPE
\begin{tabular}{|c|c|c|c|}
\hline Matching Component Values & Simulation & Prototype \\
\hline \multirow{4}{*}{ Ant1 } & Ind4 & $22 \mathrm{nH}$ & $22 \mathrm{nH}$ \\
\cline { 2 - 4 } & Ind5 & $0.4 \mathrm{nH}$ & $0.4 \mathrm{nH}$ \\
\cline { 2 - 4 } & Ind6 & $1.8 \mathrm{nH}$ & $1.8 \mathrm{nH}$ \\
\cline { 2 - 4 } & Cap7 & $12 \mathrm{pF}$ & $12 \mathrm{pF}$ \\
\hline \multirow{4}{*}{ Ant2 } & Ind8 & $1.0 \mathrm{nH}$ & $3.0 \mathrm{nH}$ \\
\cline { 2 - 4 } & Ind9 & $0.9 \mathrm{nH}$ & $1.6 \mathrm{nH}$ \\
\cline { 2 - 4 } & Cap10 & $2.4 \mathrm{pF}$ & $2.0 \mathrm{pF}$ \\
\cline { 2 - 4 } & Ind11 & $2.0 \mathrm{nH}$ & $2.9 \mathrm{nH}$ \\
\hline Ant3 & Ind12 & $1.6 \mathrm{nH}$ & $1.6 \mathrm{nH}$ \\
\hline
\end{tabular}

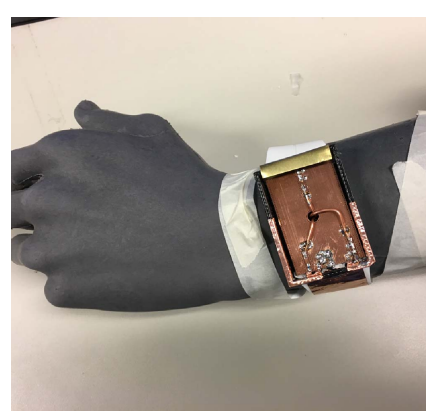

(a)

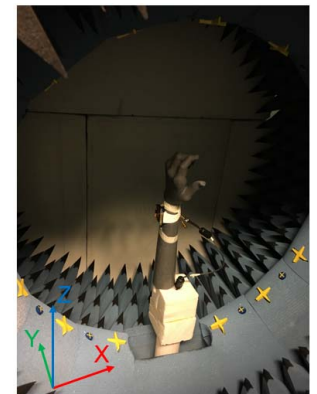

(b)
Fig. 5. (a) Wearable antenna prototype with hand phantom; (b) Installation of the prototype in Satimo StarLab Chamber for measurement of radiation gain pattern and total efficiency. 


\section{RESULTS AND DISCUSSIONS}

All simulated and measured results are based on the presence of hand and wrist phantom to mimic the real user case, which takes into account the user proximity effect on antenna performance. As it can be seen from Fig. 6, the measured reflection coefficients agree well with simulated ones. Over the operation bands of $699-862 \mathrm{MHz}, 1563$ 1605.7 $\mathrm{MHz}$ and $1710-2155 \mathrm{MHz}$, the corresponding antenna achieves as good matching level as obtained in simulations. The wideband behavior of ANT1 and ANT2 results from the efficient excitation of the fundamental mode of the whole chassis as shown in Fig. 7(a). It is seen from the current distribution, the CCE awakes the dipole mode (fundamental) of the chassis successfully and the currents distribute over the whole chassis. In other words, the antenna element makes a full use of the chassis which makes it to be an effective radiator, leading to wideband behavior. Similarly, the large bandwidth performance of ANT2 is due to the effective excitation at the corner of the planar part (in the middle of the whole chassis) where a strong electrical field distribution of the third characteristic mode takes place (see Fig. 2(c)) by an IFA (voltage source). The electrical field distribution of the fundamental mode at $780 \mathrm{MHz}$ is also provided in Fig. 7(b), indicating, again, the efficient excitation of the dipole mode (two strong electrical field distributions at two ends of the whole chassis). Moreover, it can be observed from Fig. 7(b) that the electrical field distribution is stronger at the vicinity of CCE than that at the other end. That is to say, less coupling between the chassis and wrist phantom will be obtained and higher radiation efficiency is expected.

The decoupling behavior among three antennas is also investigated. As shown in Fig. 8, the simulated and measured isolation performances indicate moderate agreements with slight decreases of coupling level in measured results. This may be caused by the lower quality factor of real components compared the ideal components. By using the matching circuits, the good isolation level is achieved by filtering out undesired response. More importantly, good isolation between ANT1 and ANT 2 as well as between ANT1 and ANT3 is also based on the fact that all characteristic modes are orthogonal to each other [15].

From the results in Fig. 9, simulated and measured total efficiencies have good agreements. To be more precise, both, the levels of simulated and measured total efficiencies, range from -11 to $-9.3 \mathrm{~dB}$, from -4.8 to $-3.8 \mathrm{~dB}$ and from -7 to -4.7 $\mathrm{dB}$ within the operation bands of $699-862 \mathrm{MHz}, 1563-$ 1605.7 MHz and 1710-2155 MHz respectively, indicating better efficiency performance compared to the previous work in [10], especially in higher frequency bands (approximately $5 \mathrm{~dB}$ improvement in bands of $1710-2155 \mathrm{MHz}$ ). In contrast to the antenna design in [10], lower coupling level is achieved in our work, resulting in these improvements in total efficiency.

To examine the performance of right hand circular polarization (RHCP) of the GPS antenna, measured radiation gain patterns of three cuts $\left(\mathrm{Phi}=0^{\circ}, \mathrm{Phi}=90^{\circ}\right.$ and $\mathrm{Theta}=$ $90^{\circ}$ ) are given in Fig. 10. From the gain patterns, it can be concluded that the designed GPS antenna has good RHCP performance at desired direction (pointing to upper space of the smartwatch to receive signals from satellite) as its gain level is higher than that of left hand circular polarization (LHCP) (more than $5 \mathrm{~dB}$ in each plane).

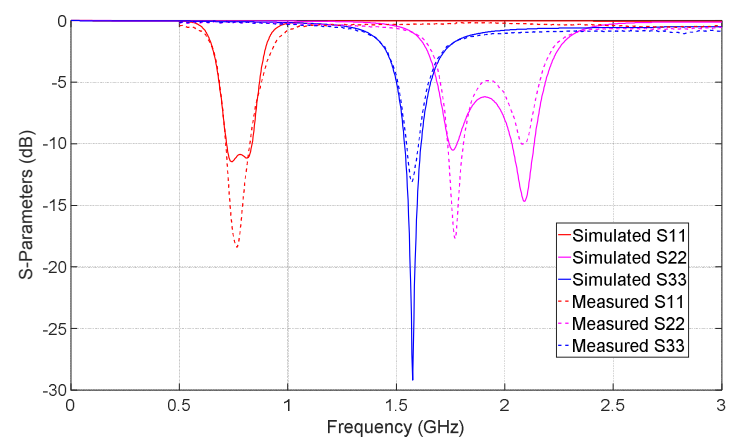

Fig. 6. Simulated and measured reflection coefficients for proposed LTE low- and mid-band antennas and GPS antenna.

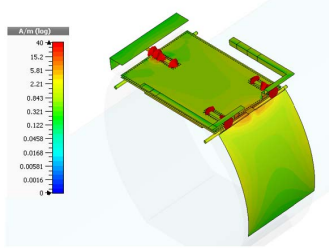

(a)

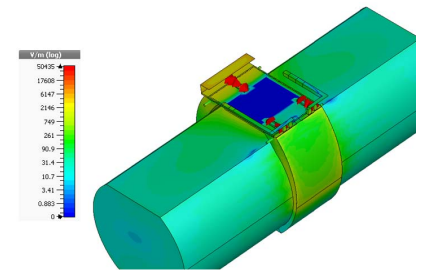

(b)
Fig. 7. (a) The current distribution and (b) the electrical field distribution of proposed wearable antenna system at $780 \mathrm{MHz}$.

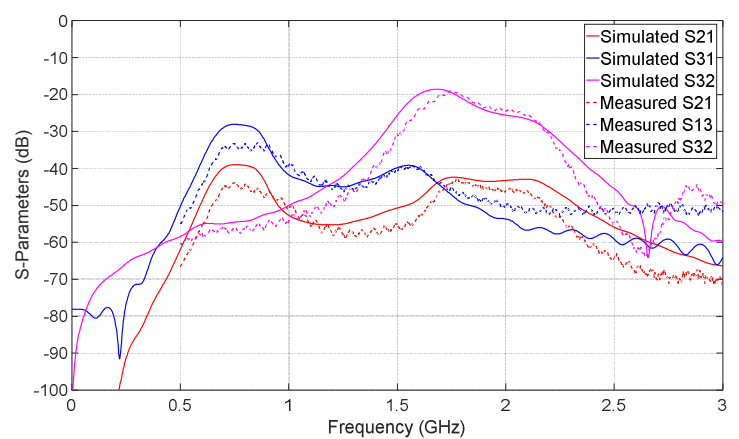

Fig. 8. Simulated and measured isolation performances between different antennas.

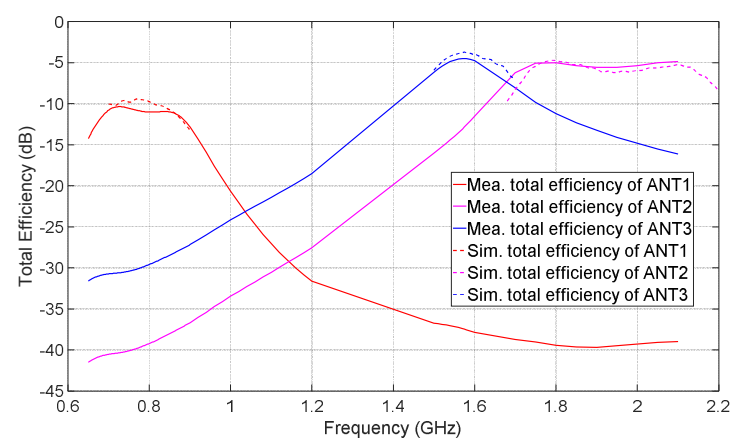

Fig. 9. Simulated and measured total efficiencies of proposed LTE lowand mid-band antennas and GPS antenna. 


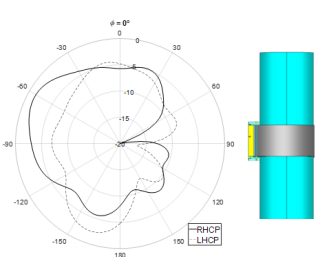

(a)

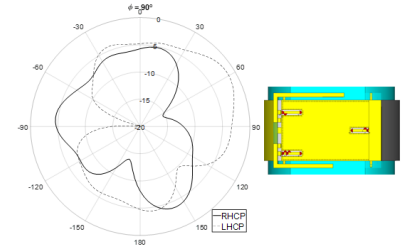

(b)

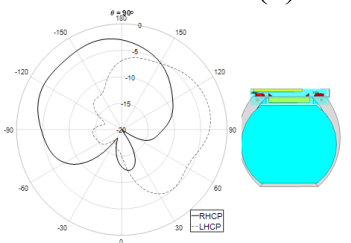

(c)

Fig. 10. The measured radiation patterns of GPS antenna (ANT3) in (a) Phi $=0^{\circ},(\mathrm{b}) \mathrm{Phi}=90^{\circ}$ and (c) Theta $=90^{\circ}$ planes

\section{CONCLUSIONS}

In this paper, we propose a new wearable three-antenna system aiming for upcoming cellular IoT applications. The antenna system is designed by using the theory of characteristic mode (TCM) to implement better performance in terms of bandwidth, isolation and efficiency, compared to previous works. In addition to covering common LTE cellular bands between $699-862 \mathrm{MHz}$ and $1710-2155 \mathrm{MHz}$, a GPS antenna with good RHCP performance is combined in the system, which has not been reported in previous works. From the results, good agreements between simulation and measurements are observed. By selecting the optimal feeding positions, the characteristic modes are successfully excited and the LTE low- and mid-band antennas can cover the required operation bands with wideband matching circuits. Meanwhile, moderate isolation performance is achieved by using orthogonal characteristic modes. Furthermore, total efficiencies are relatively good because of the efficient excitation of chassis modes. In particular, there is less coupling as the voltage feeds (CCE and IFA) are located at the casing further away from the phantom, which further improves the efficiency performance and makes it likely to be a good candidate for the future wearable cellular IoT applications.

\section{ACKNOWLEDGMENT}

This work was done in WERME research project financially supported by Hilla research program. Authors acknowledge project partnering companies for the enthusiastic and fruitful collaboration.

\section{REFERENCES}

[1] R. Ratasuk, N. Mangalvedhe, A. Ghosh and B. Vejlgaard, "Narrowband LTE-M System for M2M Communication," 2014 IEEE 80th Vehicular Technology Conference (VTC2014-Fall), Vancouver, BC, 2014, pp. 1-5.
[2] A. Díaz-Zayas, C. A. García-Pérez, Á. M. Recio-Pérez and P. Merino, "3GPP Standards to Deliver LTE Connectivity for IoT," 2016 IEEE First International Conference on Internet-of-Things Design and Implementation (IoTDI), Berlin, 2016, pp. 283-288.

[3] S. W. Su and Y. T. Hsieh, "Integrated Metal-Frame Antenna for Smartwatch Wearable Device," in IEEE Transactions on Antennas and Propagation, vol. 63, no. 7, pp. 3301-3305, July 2015.

[4] W. S. Chen, C. K. Yang and W. S. Sin, "MIMO antenna with Wi-Fi and Blue-Tooth for smart watch applications," 2015 IEEE MTT-S 2015 International Microwave Workshop Series on RF and Wireless Technologies for Biomedical and Healthcare Applications (IMWSBIO), Taipei, 2015, pp. 212-213.

[5] Chih-Hsien Wu, Kin-Lu Wong, Yuan-Chih Lin and Saou-Wen Su, "Conformal bluetooth antenna for the watch-type wireless communication device application," 2007 IEEE Antennas and Propagation Society International Symposium, Honolulu, HI, 2007, pp. 4156-4159.

[6] Di Wu, S. W. Cheung, Q. L. Li and T. I. Yuk, "Slot antenna for allmetal smartwatch applications," 2016 10th European Conference on Antennas and Propagation (EuCAP), Davos, 2016, pp. 1-4.

[7] L. J. Chu. "Physical Limitations of Omnidirectional Antennas". J. Appl. Phys.19, 1163 (1948).

[8] C. A. Balanis, "Antenna Theory, Analysis and Design". 3rd edition, 2005, Wiley.

[9] J. Chen, M. Berg, H. Y. Amin and A. Pärssinen, "Effect of smartwatch antenna location on impedance, obtainable bandwidth potential and radiation efficiency, " LAPC Conference 2017, in press.

[10] K. Zhao, Z. Ying and S. He, "Antenna designs of smart watch for cellular communications by using metal belt," 2015 9th European Conference on Antennas and Propagation (EuCAP), Lisbon, 2015, pp. 1-5.

[11] Xu Gao, Zhijun Zhang, Wenhua Chen, Zhenghe Feng, M. F. Iskander and An-Ping Zhao, "A novel wrist wear dual-band diversity antenna," 2009 IEEE Antennas and Propagation Society International Symposium, Charleston, SC, 2009, pp. 1-4.

[12] G. Li, G. Gao, J. Bao, B. Yi, C. Song and L. a. Bian, "A Watch Strap Antenna for the Applications of Wearable Systems," in IEEE Access, vol. 5, pp. 10332-10338, 2017.

[13] H. Li, Y. Tan, B. K. Lau, Z. Ying and S. He, "Characteristic Mode Based Tradeoff Analysis of Antenna-Chassis Interactions for Multiple Antenna Terminals," in IEEE Transactions on Antennas and Propagation, vol. 60, no. 2, pp. 490-502, Feb. 2012.

[14] J. Rahola and J. Ollikainen, "Optimal antenna placement for mobile terminals using characteristic mode analysis," 2006 First European Conference on Antennas and Propagation, Nice, 2006, pp. 1-6.

[15] R. Martens, E. Safin and D. Manteuffel, "Inductive and capacitive excitation of the characteristic modes of small terminals," 2011 Loughborough Antennas \& Propagation Conference, Loughborough, 2011, pp.1-4.

[16] Test plan for wireless device over-the-air performance rev. 3.6.1, CTIA Certification Standard, Nov. 2016. 